$\xi=1$ 国

\title{
BER Assessment of FBMC Systems Augmented with Different Space-Time Coding Schemes Over Diverse Channels
}

\author{
Satwinder Kaur ${ }^{1}$, Lavish Kansal ${ }^{2},{ }^{\text {Gurjot Singh Gaba }}{ }^{3}$, Mohannad A. M. Al-Ja'afari ${ }^{4}$ \\ ${ }^{1,2,3}$ Lovely Professional University, Punjab, India - 144411 \\ ${ }^{4}$ Najaf Technical Institute, Al-Furat Al-Awsat Technical University, Iraq - 31001 \\ ${ }^{1}$ satwinderkaur244@yahoo.in, ${ }^{2}$ lavish.15911@lpu.co.in, ${ }^{4}$ inj.muh@atu.edu.iq \\ *Corresponding Author $-{ }^{3}$ er.gurjotgaba@ gmail.com
}

\begin{abstract}
Diverse methodologies of encoding schemes like space-time block codes (STBC), orthogonal space-time block codes (OSTBC) \&quasiorthogonal space-time block codes (QOSTBC) are being proposed as alternatives of basic Alamouti space-time encoding scheme for multiple input multiple output (MIMO) scheme for existing wireless communication systems. Since filter bank multi-carrier (FBMC) scheme is an integral part of the $5^{\text {th }}$ generation $(5 \mathrm{G})$ cellular systems, the performance of these schemes needs to be investigated for FBMC methodology also. Alamouti and Space-time block codes are widely used in MIMO system because of their ability to achieve full diversity and the different channels are used at the receiver. In this work, we proposed different approaches for the bit error rate (BER) of Alamouti, STBC3, and STBC4 in FBMC. These approaches are based on the type of space-time encoding and number of receiving antennas being used for each space time encoding scheme for analyzing the MIMO-FBMC. Moreover, we also investigation the performance of these proposed MIMO schemes over Rayleigh and additive white Gaussian noise (AWGN) channel and compared it with the performance of BER or signal to noise ratio (SNR) of different channels.
\end{abstract}

Keywords: FBMC, BER, SNR, STBC3, STBC4, MIMO

\section{Introduction}

FBMC defines as Filter Bank Multicarrier. The first multicarrier schemes were developed in 1960 and these were based on filter bank [1]. It is a development of OFDM methodology and aims to overcome some of the issues. One of the main disadvantages of the OFDM is using a cyclic prefix. The cyclic prefix is basically a copy part of a transmitted symbol in OFDM that's attached to the start of the same symbol. The throughput of the transmission reduces due to redundancy and power is also wasted. In FBMC, at the transmitter side the multicarrier modulation function will be carried out by the IFFT and at the receiver side, the multicarrier demodulation will be the responsibility of FFT. Another disadvantage of OFDM the spectral leakage and interferences for which the weak spectral localization of the subcarriers is the main reason. Channel bank multicarrier is an improvement of OFDM. A utilization of filter banks channels that are executed, typically using digital signal processing techniques, FBMC. In OFDM systems, when carriers were modulated then side-lobes spread out on either side. With the help of filter bank system, these weak carriers are removed and give better results.

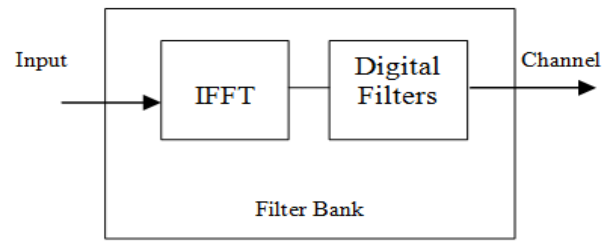

Fig. 1: Basic Diagram of Filter Bank

\section{Literature Review}

Markku Renfors et al. [2] demonstrate that the previous transmission systems depend on the OFDM technique to achieve the goals. OFDM has many negative points; the big challenges are of the cyclic prefix. Cyclic prefix causes the loss the capacity and bandwidth. It required maintaining the orthogonality among all the subcarriers. Due to the limitations of OFDM, FBMC introduced in the radio communications systems. Filter bank multicarrier can achieve higher channel capacity than OFDM because of the low spectral leakage. The spectral components are interfering to obtain very sharp frequency selectivity and they can be used very effectively. This can be done in very flexible manner.

Ari Viholainen et al. [3] give the overview of the physical layer of FBMC. The IFFT (inverse fast Fourier transform) work as the modulator and FFT (fast Fourier transform) work as the demodulator in the FBMC transmitter or receiver side respectively, and the IFFT and FFT are cascaded. Due to the cascade structure of serial to parallel conversion and parallel to serial conversion the one symbol delay has occurred at the FFT output with respect to the IFFT input symbol. When all the FFT symbols are obtained then the bank of filters are also obtained. It gives the idea about the different type of modulations like Multi Pulse Modulation isolation in time etc. In FBMC physical layer two different types of filters are used such as Synthesis Filter Bank (SFB) in OQAM Preprocessing or in modulator part and Analysis Filter Bank (AFB) in OQAM Post Processing or in Demodulator part. Saeed Afrasiabi Gorgani [5] depicts a basic idea of OFDM/OQAM with 
Synthesis and Analysis Filter Bank. In Cosine Modulated Multitone (CMT) real value (0 or 1$)$ are used and in Staggered Modulated Multi-tone (SMT) used only imaginary or complex values.

Duplexing is a two-way communication between transmitter and receiver. The forward direction is called uplink and in the reverse direction is called downlink. Yonghong Zeng et al. [6] demonstrate about FBMC duplexing. In FBMC uplink and downlink happen simultaneously and add some mechanism to separate the signals so that signals will never overlap with each other. In TDD, different time slots are used and in FDD, different frequencies (guard bands) are used for to overcome interference problems. In OFDM duplexing the signals are orthogonal to each other so that downlink and uplink signals are not overlapped with each other Loss of synchronization may also result in loss of orthogonality between the subcarriers. OFDM duplexing uplink and downlink signal don't interfere with each other because they are orthogonal. It may be destroyed by loss of synchronization. FBMC as compared to OFDM is better, FBMC uses localized prototype filter. FBMC signal is more immune to the timing errors and frequency offsets in both time and frequency domain which result in reduction of null subcarriers thereby controlling the out-of-band emissions. However, the FBMC performance also suffers from diverse parameters such as high dynamic range, high peak to average power ratio (PAPR), etc.

Qinwei He et al. [7] proposed a closed shape expressions of the bit error probabilities for both two techniques are derived and approved. The outcomes reveal that the executions of these two techniques are similar when the ideal reconstruction conditions are satisfied. In any case, the FBMC procedure has less out-of-band power leakage because of lower side projections. With an increase in the equalization complexity, the cyclic prefix enhances the bandwidth efficiency of the system. Further research can focus on the new adjustment procedure for PPN-FBMC which diminishes the multifaceted nature of moderating the signals.

An Alamouti's scheme used for Filter bank multicarrier (FBMC) to overcomes the problem of intersymbol interference (ISI). By using Alamouti's methods with FBMC modulation there are some application issues are being discussed. Due to error propagation, only interference are not effectively removes with receiver schemes. R. Zakaria et al. [18] shares own views regarding some arrangements such as STBC and space frequency block coding (SFBC). These are used for canceling the effect of interference After that Alamouti's decoding are used by interference canceller. In which basic $2 \times 1$ Alamouti's coding methods are used. Filterbank multicarrier gives the solution to overcome the problems of orthogonal frequency division multiplexing (OFDM). In FBMC filters are used so there is no need to insert guard intervals. FBMC gives the high spectral efficiency [1]. During the decoding process self- interference is not removed automatically.

FBMC modulation used two types of filters and these filters are overcome the problem of interference. Didier Le Ruyet et al. [21] elaborate the effect of defective channel state data (CSI) because of criticism interface on the execution of MU-MIMO framework utilizing Filter bank multicarrier (FBMC) balance. It depicts that the number of users is less than no. of transmit antennas then it gives better results. The bit error rate (BER), the capacity of MIMO and orthogonal frequency division multiplexing (OFDM) are similar to each other. Due to distribute of interference, results are analyzed in theoretically forms. OFDM is depended upon the number of interferes, for a given bit error rate target is set, the no. of feedback bits per channel vectors are high.

Zuleita Ho et al. [23] discussed QAM-FBMC. Specifically, Filter bank multi-carrier framework (FBMC) is considered as a promising post-OFDM challenger. Quadrature amplitude modulation (QAM) is based on FBMC (QAM-FBMC).In OFDM and FBMC comparison, FBMC has better results as analyzed as to orthogonal frequency division multiplexing, at the cost of imaginary interference makes MIMO more challenges. Ronald Nissel et al. [24] discussed the spread symbols in time and frequency domain so that with the help of this imaginary interference will remove from the system. Spreading process has low complexity due to present of Hadamard matrices. In FBMC within one transmission blocks, spreading allows restoring complex orthogonality; by this examine interference in neighboring blocks. Signal to Interference ratio can be removed by using guard time-slots. Moreover, the more ways to find the effects of time-variant channels on such spreading approach. In the end, test bed measurements depict the applicability of our FBMC based MIMO transmission scheme in real-world environments. When block-wise transmission is needed then use guard slots and interference will be decreased.

When Hundreds of antennas are used at the transmitter and then the signal undergo Rayleigh fading. Basically, large numbers of antennas are used on the transmission side and receiving side to achieve high data rate, high Capacity and reliability are also improved. Arti M.K. et al. [25] indicate the information for Alamouti's code used to achieve overall data rate for orthogonal Space-time block code (OSTBC). One advantage of OSTBC is that it has no requirement of channel state information at the transmitter for obtaining the diversity. OSTBC supports up to 32 transmitter and receiving antennas. For large dimension OSTBC is not satisfied for complex functions, it exists when the data rate may be reduced. One important thing is that in OSTBC the transmitter antenna should be less than the receiving antenna. Suppose $\mathrm{Tx}=8$ and $\mathrm{Rx}=20,16,14,12$ and 10 then result that diversity is equal to $T x * R x / 2$. In this different phase shift keying are used BPSK, QPSK, and analysis. When BPSK is used then it gives estimation about channel state information but analysis obtains the perfect channel state information through moment generating function.

\section{FBMC}

At the transmitter side used Inverse fast Fourier transform as a modulator.

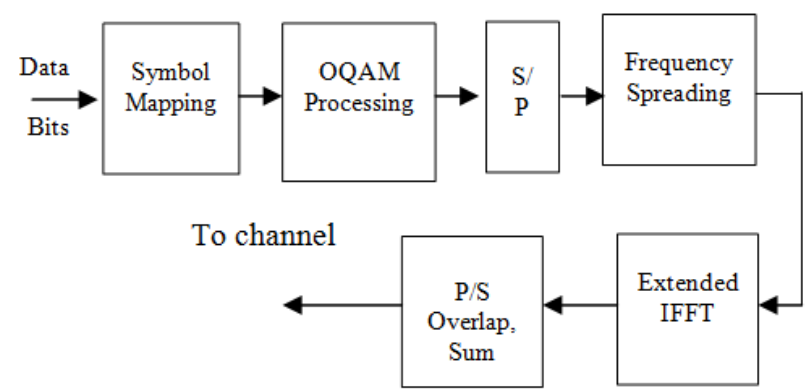

Fig. 2: FBMC transmitter

The Fig. 2 describes data bits are used as an input. The symbol mapping is used for mapping the symbols. The modulation symbol map is used to generate 16QAM modulated electrical signals and then the modulation symbol de-mapper demodulates the signals according to that which type of modulation is used. The symbol mapper's modulation type matches the mapper's modulation; the originally transmitted signal should be matched. OQAM processing has two techniques, one is OQAM pre-processing being performed at the transmitter side and other is OQAM postprocessing being performed at the receiver side. In OQAM preprocessing, the first operation is a complex to real conversion for even and odd, where the real and imaginary part of a complexvalued symbol $c_{k, n}$ are separated to form a new symbol. This means that the complex to real conversion increases the sample rate by a factor of 2 [1]. The second operation in this OQAM preprocessing is the multiplication by a sequence $\Theta_{\mathrm{k}, \mathrm{n}}$. After that 
Serial to Parallel conversion is done [3]. A serial to parallel (S/P) converter is introduced at the output of the OQAM-preprocessing and the samples data appear in the parallel form. In Frequency Spreading gives accurate equalization. Frequency spreading is a type of remote interchanges the recurrence of the transmitted signal is intentionally changed. At the block of data at the ExtendedIFFT transmitter is recovered at the output of the FFT at the receiver. A parallel-to-serial $(\mathrm{P} / \mathrm{S})$ converter is introduced at the output of the IFFT and the samples appear in the serial form. An overlap defined as when two singles are mixed with each other, but in FBMC signals are not overlap with each other.

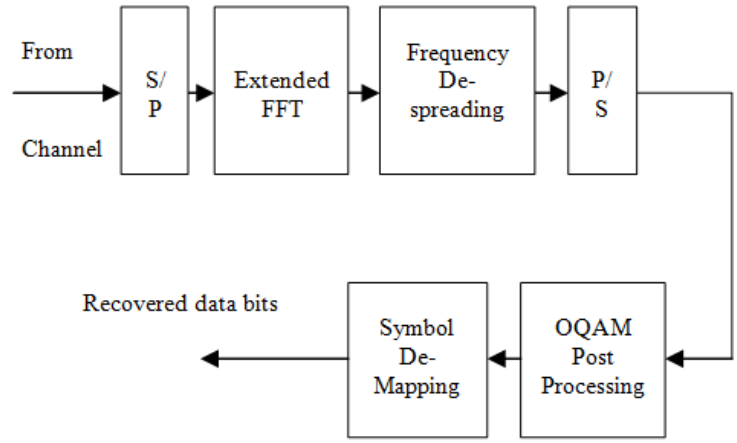

Fig. 3: FBMC receiver

In Fig. 3 shows that at the receiver side output of the transmitter of FBMC act as an input of receiver FBMC. The data is converted serial to parallel. A serial to parallel (S/P) converter is introduced at the output of the IFFT and the samples data appear in the parallel form. At the receiver side serial to parallel conversion is done, in which data sequences change from serial to parallel. In which analysis poly phase filters are used. By using this filters the process of decomposition performed by the filter bank. It can be adapted to implement the filter bank, it is just sufficient to extend the IFFT and the FFT. For each arrangement of information, the yield of the IFFT is a piece of KM tests and, since the image rate is $1 / \mathrm{M}, \mathrm{K}$ sequential IFFT yields cover in the time-space. The usage of the recipient depends on an augmented FFT, of size KM. Gotten baseband waveform is the mix of the transmitted waveform and disorder in the channel. In the modulator part, the Synthesis Filter Bank (SFB) is used. The information sources to the SFB are the offset QAM. The IFFT block basically acting the adjustment to the subcarrier frequencies. From the equipment perspective, it performs the calculations in a block preparing way. Analysis Filter Bank (AFB) is used in demodulator part. In OQAM post-processing the real to complex conversion decreases the sample rate by a factor 2 . The de-mapper modulation type matches the mapper's modulation types, where the originally transmitted signal should be recovered. In the end, at the receiver data bits are recovered.

\section{MIMO}

MIMO is defined as multiple-input multiple outputs. In which the multiple number of antennas are used at the transmitter side and at the receiver side. MIMO has the capacity to interact with various antennas at the same time which are $2 \times 2,3 \times 3$, and $4 \times 4$. Today's MIMO methods are utilized in advances systems like Wi-Fi and LTE, new strategies are under review for future benchmarks like LTE Advanced. The primary element of MIMO framework is space-time block codes. Space-Time Codes (STCs) are the codes planned for the utilization in MIMO frameworks [5]. In STCs, signs are coded in both transient and spatial areas. Here encoder used at the transmitter side and decoder at the receiver side.

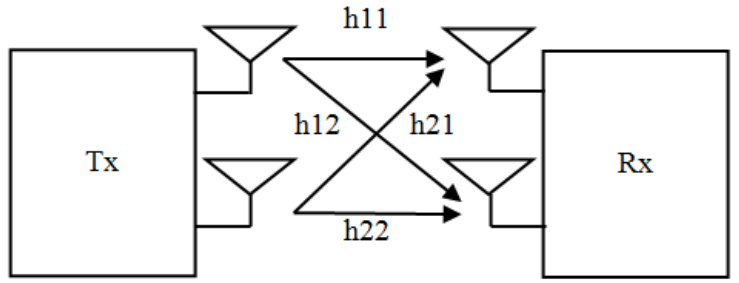

Fig. 4: Basic Diagram of MIMO

Here $h_{m n}$ represents the impulse channels. A Tx. represents the transmitter and Rx. represents receiver.

To fulfill the demand for high data rate, MIMO is combined with FBMC. MIMO is defined as Multiple Input Multiple Output. In which $\mathrm{N}$ no. transmitter signals are transferred through the different channels and received at the receiver side. To reduce fading effect between multiple data streams different MIMO channels are used such as Rayleigh and Additive White Gaussian Noise (AWGN). Another method is to minimize the effect of fading is diversity combining schemes. In this technique, the same information passes through different channels and the same information received at the receiver. If any data stream is lost then information is faded [2]. To obtain improvement through different channels the diverse methodologies are used such as Alamouti, STBC3, and STBC4. MIMO has different diverse schemes to achieve different throughput. For instance Alamouti's scheme, Space-time block coding, Omni directional space-time block coding and Quasi space-time block coding. These are explained as

Alamouti's scheme: This method is used to achieve spatial diversity for two antennas in MIMO. It presents the Alamouti's scheme coding and the space-time codes are used in it. Here discuss Alamouti's coding for the 2transmitters1receiver system $(2 \times 1)$ and 2 transmitter-2receiver system $(2 \times 2)$ [3].

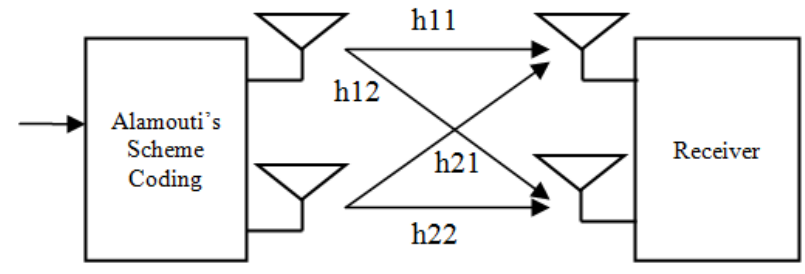

Fig. 5. Alamouti's Scheme

For 2 transmitter and receiving antennas are shown in Fig.5. Here h11, h12, h21, and h22 represent the channels from $\mathrm{N}$ transmitter to $\mathrm{N}$ receiver.

\begin{tabular}{c|cc}
\cline { 2 - 3 } Space antenna & \multicolumn{2}{c}{ Time Slot } \\
\cline { 2 - 3 } & $\mathrm{X} 2$ & $-\mathrm{X} 2^{*}$ \\
& $\mathrm{X} 1^{*}$
\end{tabular}

The basic equation of MIMO is:

$\mathrm{Y}=\mathrm{HX}+\mathrm{N}$

Where $\mathrm{y}$ is the receiving signal, $\mathrm{h}$ is the multiple channels and $\mathrm{x}$ is the transmitted symbols, $\mathrm{n}$ is noise.

- Assume two transmitter antennas as above matrix. Firstly, send two transmission symbols in first and only transmit $\mathrm{x} 1$ in first schedule and $\mathrm{x} 2$ in the next time slot.

The first receiving signal is: 


$$
\mathrm{Y} 1=\mathrm{h} 1 \mathrm{x} 1+\mathrm{h} 2 \mathrm{x} 2+\mathrm{n} 1=\left[\begin{array}{ll}
\mathrm{h} 1 & \mathrm{~h} 2
\end{array}\right]\left[\begin{array}{l}
\mathrm{x} 1 \\
\mathrm{x} 2
\end{array}\right]+\mathrm{n} 1
$$

- Alamouti's prefer to send the symbols in groups in the first schedule vacancy transmit $\mathrm{x} 1$ and $\mathrm{x} 2$ and in second-time slot send $-\mathrm{x} 2 *$ and $\mathrm{x} 1 *$

The second receiving signal is:

$$
\mathrm{Y} 2=-\mathrm{h} 1 \mathrm{x} 2 *+\mathrm{h} 2 \mathrm{x} 1 *+\mathrm{n} 2=\left[\begin{array}{ll}
\mathrm{h} 1 & \mathrm{~h} 2
\end{array}\right]\left[\begin{array}{c}
-\mathrm{x} 2 * \\
\mathrm{x} 1 *
\end{array}\right]+\mathrm{n} 2
$$

This is the simple transmission by Alamouti's space-time coding. Alamouti's have some drawback that it is not much efficient. This is an extremely exceptional STBC. It is the main orthogonal STBC that accomplishes rate -1 :

$$
\left[\begin{array}{l}
\mathrm{y} 1 \\
\mathrm{y} 2
\end{array}\right]=\left[\begin{array}{cc}
\mathrm{h} 1 & \mathrm{~h} 2 \\
\mathrm{~h} 2 * & -\mathrm{h} 1 *
\end{array}\right]\left[\begin{array}{l}
\mathrm{x} 1 \\
\mathrm{x} 2
\end{array}\right]+\left[\begin{array}{l}
\mathrm{n} 1 \\
\mathrm{n} 2
\end{array}\right]
$$

That is to express that it is the primary STBC that can fulfill its full varying qualities get without hoping to give up its data rate. Totally, this is considerable for complex change outputs. Coding rate and can't accomplish constantly most important information rate.

Space-time block coding: In STBC, multiple copies of the same signal are transmitted to exploit the spatial diversity of the wireless channel. It is an advance type of Alamouti's scheme.

Time slot $\left[\begin{array}{ccc}\text { Transmit antennas } \\ \text { S11 } & \text { S12 } \cdots & \text { S1n } \\ \text { Sm1 } & \text { S22 } & \text { Sm2 } 2 \text { S2n } \\ \text { Smn }\end{array}\right]$

$\mathrm{Y}=\mathrm{Hs}+\mathrm{n}$

STBC is an improvement of Alamouti's Space-time code [25]. MIMO systems are enabling to transmit various copies of a data stream over different radio wires. The diverse types of the data to upgrade the reliability of data trade. Space-time block coding uses both spatial and beamforming arranged qualities Space-time coding incorporates the sending of various copies data [4]. This arises multi path issues, for example, blurring and Rayleigh environment fading. When utilizing space-time block coding is used, the information stream is encoded in blocks before transmission. These information blocks are appropriated for the received output and the information is divided crosswise over time. STBC does not use for more than 2 antenna. There is some drawback that Sensitivity to channel estimation error, Delay Effects, Antenna Configurations.

Transmission by utilization of 2 antennas:

$\mathrm{H} 2=\left[\begin{array}{cc}\mathrm{x}(\mathrm{t}) 1 & \mathrm{x}(\mathrm{t}) 2 \\ -\mathrm{x}(\mathrm{t}) 2 * & \mathrm{x}(\mathrm{t}) 1 *\end{array}\right]$

Transmission by utilization of 3 antennas:

$$
\mathrm{H} 3=\left[\begin{array}{ccc}
\mathrm{x}(\mathrm{t}) 1 & \mathrm{x}(\mathrm{t}) 2 & \mathrm{x}(\mathrm{t}) 3 \\
-\mathrm{x}(\mathrm{t}) 2 & \mathrm{x}(\mathrm{t}) 1 & -\mathrm{x}(\mathrm{t}) 4 \\
-\mathrm{x}(\mathrm{t}) 3 & \mathrm{x}(\mathrm{t}) 4 & \mathrm{x}(\mathrm{t}) 1 \\
-\mathrm{x}(\mathrm{t}) 4 & -\mathrm{x}(\mathrm{t}) 3 & \mathrm{x}(\mathrm{t}) 2 \\
\mathrm{x}(\mathrm{t}) 1 * & \mathrm{x}(\mathrm{t}) 2 * & \mathrm{x}(\mathrm{t}) 3 * \\
-\mathrm{x}(\mathrm{t}) 2 * & \mathrm{x}(\mathrm{t}) 1 * & -\mathrm{x}(\mathrm{t}) 4 * \\
-\mathrm{x}(\mathrm{t}) 3 * & \mathrm{x}(\mathrm{t}) 4 * & \mathrm{x}(\mathrm{t}) 1 * \\
-\mathrm{x}(\mathrm{t}) 4 * & -\mathrm{x}(\mathrm{t}) 3 * & \mathrm{x}(\mathrm{t}) 2 *
\end{array}\right]
$$

By utilization of 4 antennas:

$$
\mathrm{H} 4=\left[\begin{array}{cccc}
\mathrm{x}(\mathrm{t}) 1 & \mathrm{x}(\mathrm{t}) 2 & \mathrm{x}(\mathrm{t}) 3 & \mathrm{x}(\mathrm{t}) 4 \\
-\mathrm{x}(\mathrm{t}) 2 & \mathrm{x}(\mathrm{t}) 1 & -\mathrm{x}(\mathrm{t}) 4 & \mathrm{x}(\mathrm{t}) 3 \\
-\mathrm{x}(\mathrm{t}) 3 & \mathrm{x}(\mathrm{t}) 4 & \mathrm{x}(\mathrm{t}) 1 & -\mathrm{x}(\mathrm{t}) 2 \\
-\mathrm{x}(\mathrm{t}) 4 & -\mathrm{x}(\mathrm{t}) 3 & \mathrm{x}(\mathrm{t}) 2 & \mathrm{x}(\mathrm{t}) 1 \\
\mathrm{x}(\mathrm{t}) 1 * & \mathrm{x}(\mathrm{t}) 2 * & \mathrm{x}(\mathrm{t}) 3 * & \mathrm{x}(\mathrm{t}) 4 * \\
-\mathrm{x}(\mathrm{t}) 2 * & \mathrm{x}(\mathrm{t}) 1 * & -\mathrm{x}(\mathrm{t}) 4 * & \mathrm{x}(\mathrm{t}) 3 * \\
-\mathrm{x}(\mathrm{t}) 3 * & \mathrm{x}(\mathrm{t}) 4 * & \mathrm{x}(\mathrm{t}) 1 * & -\mathrm{x}(\mathrm{t}) 2 * \\
-\mathrm{x}(\mathrm{t}) 4 * & -\mathrm{x}(\mathrm{t}) 3 * & \mathrm{x}(\mathrm{t}) 2 * & \mathrm{x}(\mathrm{t}) 1 *
\end{array}\right]
$$

\section{Simulation Results}

The simulation results for illustrating the impact of using MIMO systems on the BER vs SNR performance of FBMC system are presented in this session. In the simulation work, AWGN and Rayleigh channels are used for analyzing the performance of FBMC system augmented with MIMO system. In MIMO-FBMC, different diversity combining techniques are used to analyze the performance of the system.

\section{A) AWGN augmented FBMC:}

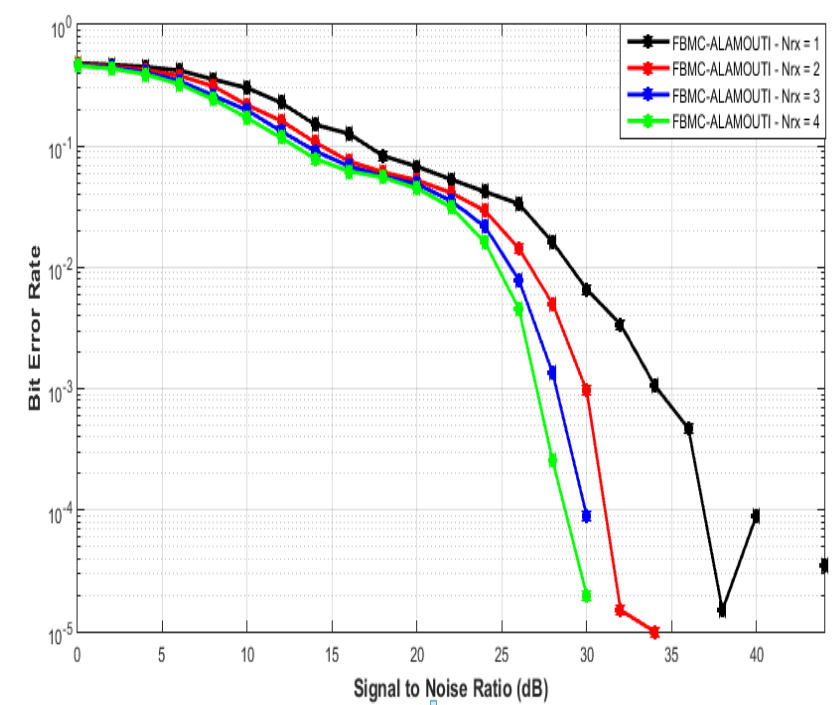

Fig. 6(a): SNR vs BER performance of FBMC-Alamouti in AWGN channel

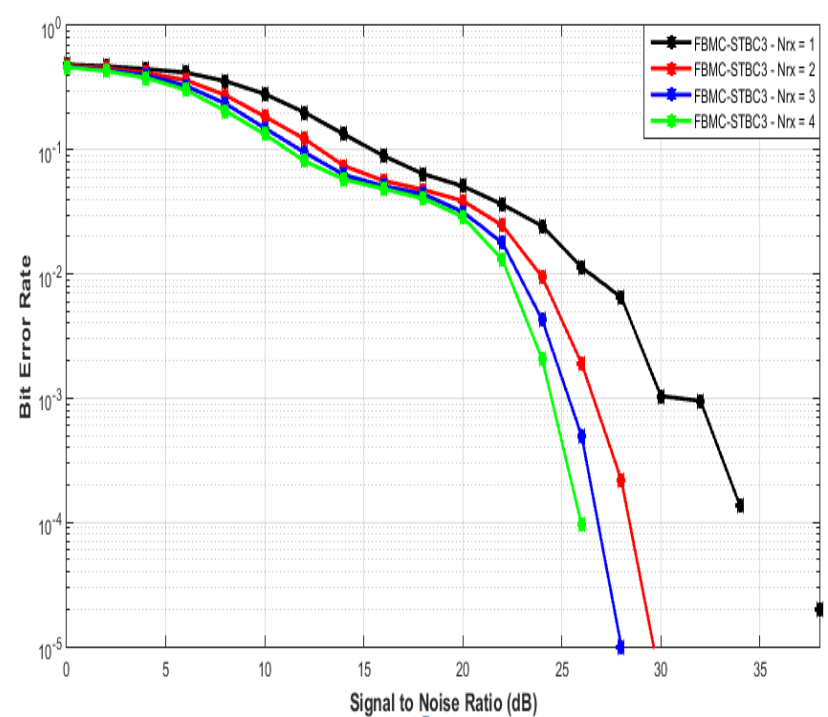

Fig. 6(b): SNR vs BER performance of FBMC-STBC3 in AWGN channel 


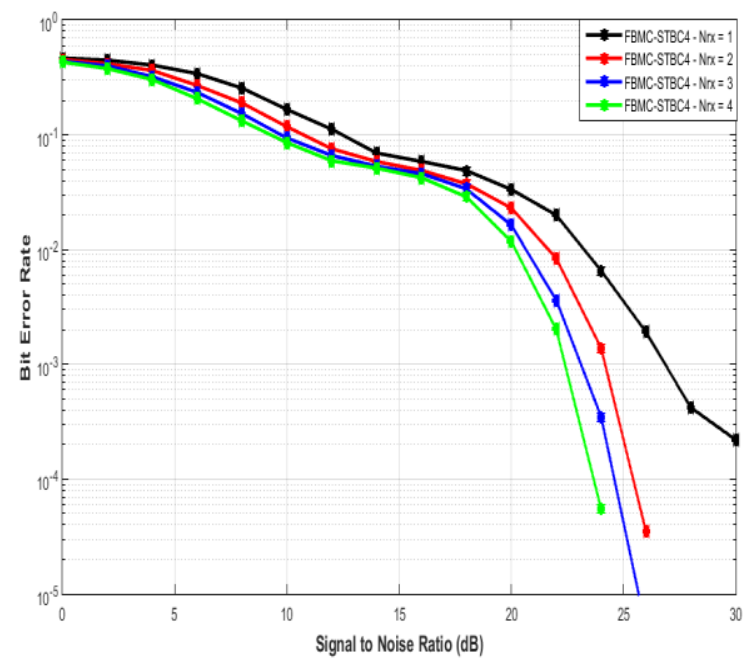

Fig. 6(c): SNR vs BER performance of FBMC-STBC4 in AWGN channel

Table 1: SNR requirement for FBMC-MIMO in AWGN channel to achieve desired BER

\begin{tabular}{|c|c|c|c|c|}
\hline \multirow{2}{*}{$\begin{array}{l}\text { Diversity tech- } \\
\text { niques }\end{array}$} & \multicolumn{4}{|c|}{$\begin{array}{l}\text { SNR (dB) required to achieve a BER of } 10^{-3} \text { for } \\
\text { different receiving antennas }\end{array}$} \\
\hline & $N_{r x}=1$ & $N_{r x}=2$ & $N_{r x}=3$ & $N_{r x}=4$ \\
\hline Alamouti & 34 & 30 & 28 & 27 \\
\hline STBC3 & 30 & 27 & 25 & 23.5 \\
\hline STBC4 & 27 & 24 & 23 & 22 \\
\hline
\end{tabular}

In Fig. 6 (a-b\&c) FBMC- Alamouti, FBMC-Space-Time Block Code-3 (STBC3)\& FBMC-Space-Time Block Code-4 (STBC4) diversity technique are used with varying number of receiving antennas $\mathrm{N}$ over AWGN channel. Here, the number of receiving antennas are varied from 1 to 4 i.e. $\mathrm{N}=1,2,3$ and 4 . By using different antennas at the receiver side, BER performance of FBMC is showing a varying effect. When the number of receiving antennas are increasing, the Bit Error Rate keeps on decreasing and thus this system would provide better Bit Error Rate performance with respect to a specified value of SNR. The performance of FBMC-STBC4 is better in case of AWGN channel, in comparison to Alamouti space-time encoding and OSTBC3 space-time encoding.

\section{B) Rayleigh augmented FBMC:}

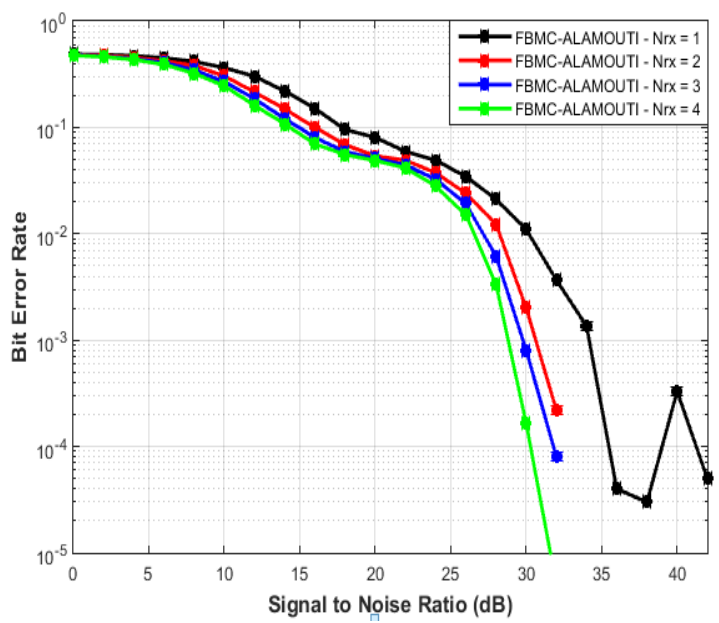

Fig. 7(a): SNR vs BER performance of FBMC-Alamouti in Rayleigh Chan.

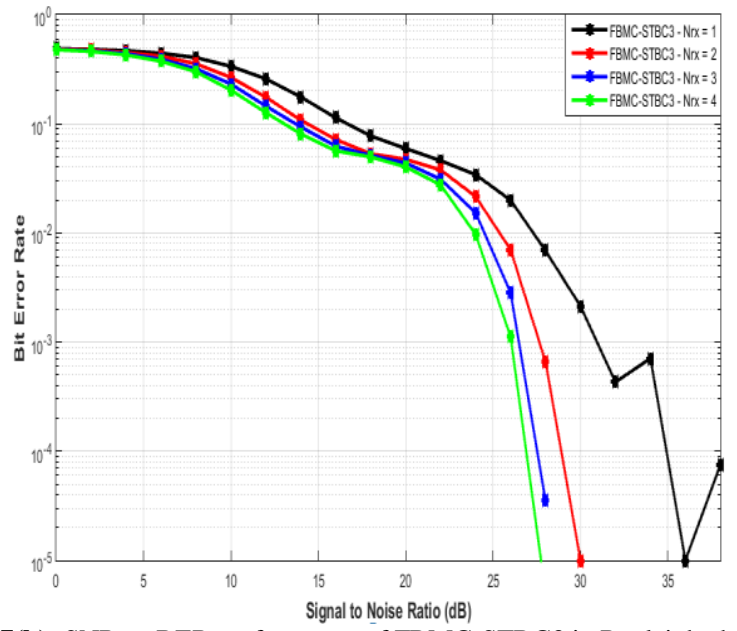

Fig. 7(b): SNR vs BER performance of FBMC-STBC3 in Rayleigh channel.

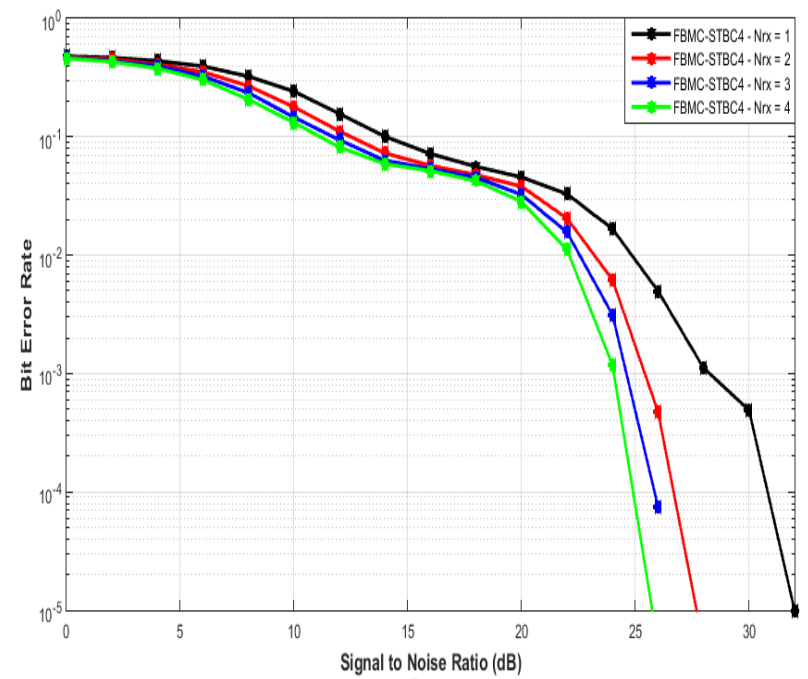

Fig. 7(c): SNR vs BER performance of FBMC-STBC4 in Rayleigh channel

Table 2: SNR requirement for FBMC-MIMO in Rayleigh channel to achieve desired BER

\begin{tabular}{ccccc}
\hline \multirow{2}{*}{$\begin{array}{c}\text { Diversity } \\
\text { techniques }\end{array}$} & \multicolumn{4}{c}{$\begin{array}{c}\text { SNR (dB) required to achieve a BER of 10 } \\
\text { different receiving antennas }\end{array}$} \\
\cline { 2 - 5 } & $\mathbf{N}_{\mathrm{rx}}=\mathbf{1}$ & $\mathbf{N}_{\mathrm{rx}}=\mathbf{2}$ & $\mathbf{N}_{\mathrm{rx}}=\mathbf{3}$ & $\mathbf{N}_{\mathrm{rx}}=\mathbf{4}$ \\
\hline Alamouti & 34.5 & 31 & 29.5 & 28 \\
STBC3 & 31.5 & 27 & 26.5 & 25 \\
\hline STBC4 & 28 & 25.5 & 24.5 & 24
\end{tabular}

In Fig. 7 (a-b\&c) FBMC- Alamouti, FBMC-Space-Time Block Code-3 (STBC3)\& FBMC-Space-Time Block Code-4 (STBC4) diversity technique over Rayleigh channel are deonstrated with varrying number of receiving antennas. Here, the number of receiving antennas are varied from 1 to 4 i.e. $\mathrm{N}=1,2,3$ and 4 . By using different antennas at the receiver side, BER performance of FBMC varrying. When the number of receiving antennas are increasing, the Bit Error Rate keeps on decreasing and thus this system would provide better Bit Error Rate performance with respect to a specified value of SNR. The performance of FBMC system augmented with Alamouti space-time encoding, OSTBC3, and STBC4 is better in case of AWGN channel in comparison to Rayleigh channel, as in case of Rayleigh channel no line of sight path is available in between the transmitter and receiver, moreover, the effect of multipath fading also comes in to play in case of rayleigh fading channel. 
Here, from graphs 6 and 7 observed that using STBC3 and STBC4 diversity techniques, STBC4 gives better performance than STBC3 in case of AWGN rather than Rayleigh channels.

\section{Conclusion}

In MIMO-FBMC system, the use of multiple antenna's for different technique such as Alamouti, STBC3, and STBC4 results in significant improvement in BER performance of the system over AWGN and Rayleigh channel. The simulation results depict that when the number of receiving antennas is increasing, the BER keeps on decreasing. The use of multiple antennas results in decrease in SNR requirement to achieve a desired BER by 4-7 dB. Moreover, the BER performance is improving on increasing the transmitting as well as receiving antennas, as BER for STBC4 augmented FBMC is less than the BER of Alamouti augmented FBMC.

\section{References}

[1] Viholainen A, Ihlainen T, Stitz TH, Renfors M \&Bellanger M, "Prototype filter design for filter bank based multicarrier transmission", $17^{\text {th }}$ European Signal processing conference, pp. 1359-1363, Glasgow, August-2009.

[2] Renfors M, Siohan P, Boroujeny BF\& Bader F, "Filter Banks for Next Generation Multicarrier Wireless Communications", EURASIP Journal on Advances in Signal Processing, vol. 2010, pp. 2 , 2010, doi: 10.1155/2010/314193

[3] Viholainen A, Bellanger M \&Huchard M, "FBMC Physical Layer: a primer", Physical Layer for Dynamic Spectrum Access and Cognitive Radio, pp. 1-31, Paris, June-2010.

[4] Ruyet DL, Zakaria R \& Ozbek B, "On precoding MIMO-FBMC with imperfect channel state information at the transmitter", $11^{\text {th }} I n$ ternational Symposium on Wireless Communications Systems (ISWCS),pp. 808-812,Barcelona, Spain, 2014, doi: 10.1109/ISWCS.2014.6933464

[5] Gorgani SA, "An Introduction to OFDM/OQAM", pp.1-18, Berlin May-2014https://arxiv.org/abs/1707.08934

[6] Zeng Y, Liang YC, Chia MW \& Edward CY, "FBMC Duplexing: Advantages and Problems", 10th International conference Information Communications and Signal Processing, pp. 1-5,Singapore, April-2015, doi: 10.1109/ICICS.2015.7459955

[7] Q. H \&Schmeink A, "Comparison and evaluation between FBMC and OFDM systems", $19^{\text {th }}$ international ITG workshop on smart antennas (WSA), pp. 1-7, Germany, March-2015.

[8] Xu M, Zhang J, Lu F, Wang J, Cheng L, Cho H, Khalil MI, Guidotti D \& Chang GK., "FBMC in Next-Generation Mobile Front haul Networks with Centralized Pre-Equalization", IEEE Photonics Technology Letters, Vol. 28, Issue 18, pp. 1912-1915, September2016, doi: 10.1109/LPT.2016.2575060

[9] Chunkath J, Arjun SS, Sheeba VS \& Raj A, "Performance Improvement Of Multicarrier Systems Using Wavelet Filter Banks", International Conference on Emerging Trends in Engineering, Science and Technology, Vol. 24, pp. 775-781,Thrissu, 2016, doi: 10.1016/j.protcy.2016.05.087

[10] Mattera D, Tandaa M \&Bellanger M, "Filter Bank Multicarrier with PAM Modulation for Future Wireless Systems", Signal processing conference, Vol. 120, pp. 594-606, France, March-2016, doi: $10.1016 /$ j.sigpro.2015.09.035

[11] Abdullahi AB, "Performance Evaluation of MIMO System Using LTE Downlink Physical Layer", IEEE SAI Computing Conference, Vol. 978, pp.4673-8460, London UK, July-2016, doi: 10.1109/SAI.2016.7556053

[12] Rabiei P \& Al-Dhahir N, “A New Full-Rate Full-Diversity STBC for 2 TX with Low Complexity ML Decoding”, IEEE Sarnoff Symposium, USA,2007, doi: 10.1109/SARNOF.2007.4567356

[13] SeungAhn K, "Performance Analysis of MIMO-MRC System in the Presence of Multiple Interferers and Noise over Rayleigh Fading Channels", IEEE Trans. on Wireless Commun., Vol. 8, Issue 7, pp. 3727- 3735, July- 2009, doi: 10.1109/TWC.2009.080940

[14] Tiwari K, Jain A \& Charhate SY,"BER Analysis of Nakagami-m Channels with different Modulation Techniques and Transmit Diversity", Proc. of IEEE International Conference (ICM2CS'09), pp.1-3, New Delhi, December-2009.
[15] Lozano A \& Jindal N, "BER performance of multi user scheduling for MIMO-STBC and MIMO-OFDM broadcast network with imperfect CSI", IEEE Transactions on Wireless Communications, Vol. 9, Issue 1, pp. 186-197, India, 2010, doi: 10.1109/SPIN.2017.8049917

[16] Kumar NS, Foschini GJ, Golden GD \& Valenzuela R A, "Bit Error Rate Performance Analysis of ZF, ML and MMSE Equalizers for MIMO Wireless Communication Receiver", European Journal of Scientific Research, Vol. 59, Issue 4, pp. 522-532, 2011.

[17] Singh RP, "BER performance of MIMO- STBC system in Nakagami-m fading channel with transmit diversity schemes", VSRDinternational journal of electrical and electronics and communication engineering, Vol. 2, pp. 448-455, 2012, doi: 10.1109/ICHPCA.2014.7045307

[18] Zakaria R \&Ruyet DL, "On interference cancellation in Alamouti coding scheme for filter bank based multicarrier systems", The Tenth International Symposium on Wireless Communication Systems, pp. 1-5, Germany, 2013.

[19] Caus M \&Neira AIP, "Multi-stream transmission in MIMO-FBMC systems", IEEE international conference on Acoustics, Speech Signal processing (ICASSP), pp. 5041-5045, Canada, May-2013, doi: 10.1109/ICASSP.2013.6638621

[20] Dholakia PM, Kumar S, Vithlani CH \& Solanki M, "On performance analysis of OSTBC-MIMO systems for audio [mp3] transmission in Rayleigh fading environments", IEEE International conference on Advances in Communication and Computing Technologies, pp.1-7, Mumbai, India,2014, doi: 10.1109/EIC.2015.7230700

[21] Ruyet DL, Zakaria R \& Ozbek B, "On precoding MIMO-FBMC with imperfect channel state information at the transmitter", $11^{\text {th }} \mathrm{In}$ ternational Symposium on Wireless Communications Systems (ISWCS),pp. 808-812,Barcelona, Spain,2014, doi 10.1109/ISWCS.2014.6933464

[22] Winn KC, Han P, Sewaiwar A \&Chung YH, "Quasi-Orthogonal Space-time Block Code (QOSTBC) for Full-Rate Massive MIMO with up to 32 Antennas",IEEE conference U-GIT, pp.1-6, South Korea, July-2014

[23] Ho Z, Kim K, Kim C, Yun YH, Cho YH \&Seol JY, "A QAMFBMC Space-Time Block Code System with Linear Equalizers", IEEE Globecom Workshops (GC Wkshps), pp.1-5, San Diego, CA, December-2015, doi: 10.1109/GLOCOMW.2015.7414208

[24] Nissel R \& Rupp M, "Enabling Low-Complexity MIMO in FBMCOQAM", IEEE Globecom Workshops (GC Wkshps), Washington, 2016 , 10.1109/GLOCOMW.2016.7848888

[25] Member AMK, "OSTBC Transmission in Large MIMO Systems", IEEE Communications Letters, Vol. 20, Issue 11, pp. 2308 - 2311 November-2016, doi: 10.1109/LCOMM.2016.2597229 